\title{
A Case Series of Inferior Vena Cava Filter Implantation in Three COVID-19 Patients with Deep Venous Thrombosis
}

\author{
chuanqi cai ${ }^{1}$, Yi Guo ${ }^{1}$, Yun You $^{1}$, Fei Cai ${ }^{1}$, Mingxing Xie ${ }^{1}$, Yiqing Li $^{1}$, and Weici Wang ${ }^{1}$ \\ ${ }^{1}$ Wuhan Union Hospital
}

July 24, 2020

\begin{abstract}
Three COVID-19 cases were simultaneously confirmed as deep venous thrombosis (DVT). Ultrasound-guided IVCF implantation is an effective method that can be considered as a precautionary strategy for preventing lethal pulmonary embolism occurrence, especially for critical DVT-COVID-19 patients who are not suitable to be transferred to a routine X-ray operation room.
\end{abstract}

\section{Introduction}

Since December 8, 2019, an epidemic of coronavirus disease 2019 (COVID-19) has spread rapidly. There are more than 3,672,238 COVID-19 patients worldwide, with 254,045 deaths having been reported to date ${ }^{1}$. Deep venous thrombosis (DVT) has been reported to be as high as $31 \%$ in intensive care unit (ICU) patients with COVID-19 infections ${ }^{2}$. Moreover, fatal pulmonary embolization (PE) has been reported to be as high as $20.6 \%$ in severe ICU COVID-19 patients ${ }^{3}$. Inferior vena cava filter (IVCF) implantation is the recommended precautionary strategy for fatal PE among DVT patients ${ }^{4}$. IVCF implantation is generally performed under X-ray or color ultrasound (US) guidance ${ }^{4}$. Ultrasound-guided IVCF implantation at the bedside is however more convenient and carries a lower risk of infection ${ }^{5}$. There is currently no guidance with respect to the preferred method of implantation during the current COVID-19 pandemic. Here, we present three confirmed cases of DVT-COVID-19 patients. All of them had reached IVCF implantation intervention indications. We performed IVCF implantation for the first patient under digital subtraction angiography (DSA) in the DSA operating room and for the other two, under bedside ultrasound in the COVID-19 isolation ward. All patients or their representatives have consented to the publication of details and images pertaining to the cases.

\section{Case presentation}

\section{CASE 1}

A 61-year-old woman patient (PADUA index: 4, Caprini index: 5) experienced 7 days of respiratory distress. She was confirmed COVID-19 positive thanks to a real-time reverse transcription polymerase chain reaction (rRT-PCR) test. After admission, high flow nasal cannula (HFNC) and supportive care were performed immediately. At day 4, she complained of fatigue of the right lower limb. Due to the low oxygen saturation, a cranial computerized tomography (CT) scan was not performed immediately. At day 6, a bedside chest X-ray was performed, as shown in Fig. 1A . At day 8, there was obvious edema of the right lower extremity. Consequently, the patient was scheduled for color ultrasound (US) detection to screen for deep venous thrombosis (DVT). The scan confirmed that there was fresh thrombosis in the right femoral vein. She was 
anticoagulated with enoxaparin sodium (4000U, q12 h). At day 10, the patient became drowsy and had evidence of right limb hemiplegia. A cerebral haemorrhage may have been the reason for these symptoms, a leading cause of morbidity in COVID-19 patients ${ }^{6}$. Anticoagulation was consequently withheld indefinitely and recommended implantation of an IVCF to prevent fatal PE occurrence. A CT scan of the chest at Day 10 had showed typical COVID-19 features in the lungs (Fig. 1B ). She was transported to the digital subtraction angiograph (DSA) operation room for IVCF implantation. After a successful Seldinger puncture of the left femoral vein, a permanent VenaTech ${ }^{\circledR}$ LP IVC filter (B. Braun, Melsungen, Germany) was placed underneath the right renal vein, as shown in Figs. 1C-1D. Antibiotic and supportive therapies were also optimised. A repeat chest X-ray on day 13 (Fig. 1E ) had showed signs of improvement compared to day 6 . She was followed up regularly with her blood work (Table 1 ). C-reactive protein (CRP), neutrophil count, D-dimer, and high-sensitivity Troponin I (hsTNI) levels were all on the rise. The lymphocyte count was however improving. This together with hsTNI normalised at day 21. CT scan of the chest at day 23 (Fig. $1 \mathrm{~F})$.

She was eventually asymptomatic and discharged at day 28 . This patient was the first case to receive DSAguided IVCF implantation in our COVID-19 therapy centre. The DSA operation room and equipment was meticulously disinfected to safeguard the health of patients making use of the service in the future.

\section{CASE 2}

A 70-year-old male patient (PADUA index: 5, Caprini index: 7) was confirmed as having COVID-19 disease when he received a routine preoperative pulmonary CT scan due to arterial and venous thrombosis in the right extremity. After 25 days of chronic ischemia, there was dry gangrene of his right thigh (Fig. 2A ). On admission to hospital, physical examination showed that his right lower limb appeared swollen and tender; displayed cyanotic change with a diminished dorsalis pedis pulse. Color US detection determined that he was suffering from a right iliac arterial and venous embolism. A series of other poorly prognostic symptoms, such as left toe ischemia (Fig. 2B ) and left lower extremity inter-muscular venous thrombosis were also found. His abdominal aorta and left iliac arteries remained unobstructed. His only past medical history of note was surgery for lung cancer four years previously.

Initial laboratory tests (Table 2 ) showed a high CRP $(65.85 \mathrm{mg} / \mathrm{L})$, white blood cell $(9.83 \mathrm{G} / \mathrm{L})$, neutrophil count $(7.49 \mathrm{G} / \mathrm{L})$, alanine transaminase (ALT) $(127 \mathrm{U} / \mathrm{L})$, aspartate transaminase (AST) $(119 \mathrm{U} / \mathrm{L})$ levels, D-dimer $(6.55 \mathrm{ug} / \mathrm{mL})$ and fibrinogen (FIB) $(5.6 \mathrm{~g} / \mathrm{L})$. Creatine kinase-MB (CKMB) and hsTNI levels were normal. His oxygen saturation (SPO2) was $89 \%-91 \%$ for room air and $96 \%$ on high-flow nasal cannula oxygen therapy (HFNC). He was unable to undergo revascularisation surgery to salvage the right lower limb. He was scheduled for right thigh amputation surgery following IVCF implantation (Figs. 2C-2E ). At day 1, he received anticoagulation (enoxaparin, 4000U, s.c), an antibiotic and supportive therapies.

At day 2, this patient was scheduled for implantation of a retrievable Cook Celect IVCF (William Cook, Europe) for preventing acute pulmonary embolism (PE) during the amputation surgery. The intervention was performed with bedside US on the isolation ward. This confirmed patency of the inferior vena cava, left iliac vein, and femoral vein. It also assured that percutaneous US guided IVCF implantation is feasible (Fig. 2C ). After local anesthesia and successful Seldinger puncture of the left femoral vein, the filter sheath was visually inserted into the left femoral vein through one 6 Fguide sheath. Next, we injected $10 \mathrm{~mL}$ diluted SonoVue US contrast agent (Bracco, Milan, Italy) through the filter sheath to adjust the catheter tip location. The tip of the filter sheath was anchored $1 \mathrm{~cm}$ below the right renal vein (Fig. 2D ). The filter was delivered into the catheter and deployed according to the manufacturer's recommendations (Fig. 2E ). IVCF was deployed successfully. The patient was transferred to a disinfected theatre and proceeded with right thigh amputation surgery. Post-operatively, he was transferred to the isolated intensive care unit (ICU). Severe bilateral pneumonia was confirmed on a bedside chest X-ray on day 3 (Fig. 2F ). The IVCF position remained unchanged (Fig. 2F ). His D-dimer had progressively decreased from the initial value of $6.55 \mu \mathrm{g} / \mathrm{mL}$ to $2.85 \mu \mathrm{g} / \mathrm{mL}$ at day 7 after continuous anticoagulation therapy (enoxaparin, 4000U, s.c, 
q12 h). At day 9, he suffered an acute cardiac event with elevated hsTNI (10025.40 ng/L). He was treated medically for acute coronary syndrome. Supportive management was also optimised. A chest X-ray again confirmed severe bilateral pneumonia with no shit in the IVCF. At day 10, the creatine kinase-MB (CKMB) $(262.70 \mathrm{ng} / \mathrm{mL})$ and hsTNI (>50000.00 ng/L) levels kept increasing despite aggressive medical therapy. The patient eventually passed away on day 11 (Table 2 ).

\section{CASE 3}

A 40-year-old man (PADUA index: 4, Caprini index: 8) was confirmed as having severe COVID-19 by a rRT-PCR test. A routine CT scan on day 3 also confirmed typical COVID-19 radiographic manifestations, as shown inFigs. 3A-3B . On admission, this patient claimed to be in good physical health. He was however complaining of partial paralysis and an uncomfortable feeling of his left lower limb at day 4. A bedside X-ray showed compressed fractures in the 12th thoracic vertebrae (T12) and the 1st lumbar (L1) vertebrae (Figs. 3C-3D ). Physical examination showed that his left lower limb was mildly swollen, the skin temperature and skin color were normal, and the doralis pedis pulse was palpable. Bedside US scanning showed fresh venous thrombosis in the left iliac vein.

At the time of consultation, the patient experienced continuous lower limb paralysis. It was decided to proceed with internal fixation surgery of both T12 and L1 fractures. Iliac vein thrombosis however increased the risk of PE during orthopaedicsurgery ${ }^{7}$. A pre-operative IVCF could substantially decrease this risk. Initial laboratory tests (Table 3 ) showed higher levels of white blood cell count (11.89 G/L) and neutrophil count $(9.35 \mathrm{G} / \mathrm{L})$. His D-dimer increased within 24 hours from 1.26 to $1.70 \mu \mathrm{g} / \mathrm{mL}$. The hsTNI and CKMB levels were normal.

At day 5, he was scheduled for pre-operative implantation of a retrievable Cook Celect IVCF (William Cook, Europe). His IVCF intervention was also performed with the aid of beside US on the isolation ward (Figs. 3E-3G ), following the similar procedures reported for patient 2. At day 6 , he underwent orthopaedic surgery and was transferred to his isolation post-operatively. At day 9, an X-ray confirmed that his IVC filter had not shifted (Figs. 3H-3I ). At day 18, another CT scan was performed which showed complete normalisation of lung parenchyma. He recovered well from COVID-19 and was subsequently discharged.

\section{Discussion}

Venous thromboembolism (VTE), comprising deep venous thrombosis (DVT) and pulmonary embolism $(\mathrm{PE})$, is a common and serious complication predominantly occurring in hospitalized patients. The estimated incidence of a first acute VTE is 0.7 to 1.4 per 1000 person-years and is mostly observed in patients older than 55 years $^{8}$. Marik et al. reported an $11.7 \%$ incidence of DVT in a group of medical ICU patients ${ }^{9}$. The lack of resources limited our ability to systematically perform US screening for each COVID-19 patient with a VTE risk factor. The real morbidity and mortality of VTE in COVID-19 patients needs to be investigated further.

The treatment of DVT and PE is nonspecific. Anticoagulant and thrombolytic therapies are essential strategies. However, for hemorrhagic or preoperative patients who are suffering from DVT simultaneously, IVC filter insertion could be an effective intervention for preventing lethal PE.

Our report describes three severe COVID-19 patients also presenting with DVT disease. All of them had reached IVCF implantation intervention criteria to prevent acute PE. There are however no recommendations on the best IVCF implantation strategy in the latest COVID-19 practice guidelines. ICVF implantation is normally performed under digital subtraction angiography (DSA) guidance, as presented in case 1. Severe COVID-19 patients however often present with hypoxaemia, requiring continuous HFNC and ECG monitoring. These unstable features would not allow transfer of these patients to the DSA room. Transferring 
patients from isolation wards would also increase the likelihood of cross-infection in the hospital. Disinfection of the DSA room would have a significant impact on medical and non-medical resources, together with manpower. The team thus opted for US guided IVCF implantation for both remaining cases.

Up to the point of submission, we believe that this is the first bedside US-guided IVCF implantation reported case in severe COVID-19 patients on the isolation ward. We would like to summarise our experience pertaining to thrombosis prevention and treatment in severe and critical COVID-19 patients as follows:

1. More attention should be paid to the prevention and treatment of DVT and PE, in spite of the current shortage of critical medical resources;

2. For severe and critically ill patients, primary preventive strategies with stockings and/or drugs should be encouraged. Physicians should closely monitor biomarkers of hypercoagulation. There should also be increased awareness about the possibility of neurovascular compromise in the extremities. Routine colour US scans may be necessary for COVID-19 inpatients;

3. During the current COVID-19 epidemic, an US-guided IVCF implantation on the isolation ward may be considered in patients who fulfil criteria for implantation;

4. Full preparation is needed to achieve bedside IVCF implantation: pre-assessment of the feasibility of a percutaneous approach via the femoral vein; assessment of whether the inferior vena cava is unobstructed; evaluation of whether there is vena cava variation; fasting for 12 hours before surgery (giving an ordinary enema 1 hour before surgery is another alternative choice). Vascular surgeons need to take level 3 protective measures before entering the isolation ward and strictly follow protective protocol to avoid contamination.

In conclusion, COVID-19 positive patients with abnormal signs and symptoms in the extremities should be monitored closely. A colour US scan is recommended for severe and critical COVID-19 patients with a high PADUA index and/or Caprini index. If the patients have reached an IVCF implantation indication, we strongly recommend opting for an US-guided bedside IVCF implantation strategy on the isolation ward instead of the conventional DSA method.

\section{Declarations}

\section{Ethics approval and consent to participate}

This study was approved by the Ethics Committee of Union hospital Wuhan, China. The informed consents of the participants were acquired.

\section{Competing interests}

The authors declare that they have no competing interests.

\section{Funding}

This research was supported by National Natural Science Foundation of China (NO.81873529) to W.W.

Acknowledgements The authors deeply thank all colleagues working in Union Hospital for supporting and collecting the valuable COVID-19 patients' data.

\section{Author contribution}

C.C.: contributed to guarantor, literature search, manuscript editing, manuscript preparation, interpretation of data, and study design. Y.G.: contributed to design, interpretation of data, literature search, manuscript preparation. Y.Y.: contributed to manuscript preparation, interpretation of data, and manuscript review. F.C.: contributed to manuscript preparation. M.X.: contributed to interpretation of data, and manuscript review. Y.L.: contributed to study design and manuscript review. W.W.: contributed to guarantor, literature 
search, manuscript review, manuscript editing, manuscript preparation, interpretation of data, and study design. All authors read and approved the final version to be published.

\section{Reference}

1. Organization WH. Coronavirus disease (COVID-2019) situation reports. 2020;2020.

2. Klok FA, Kruip MJHA, van der Meer NJM, Arbous MS, Gommers DAMPJ, Kant KM, Kaptein FHJ, van Paassen J, Stals MAM, Huisman MV and Endeman H. Incidence of thrombotic complications in critically ill ICU patients with COVID-19. Thromb Res . 2020:S0049-3848(20)30120-1.

3. Poissy J, Goutay J, Caplan M, Parmentier E, Duburcq T, Lassalle F, Jeanpierre E, Rauch A, Labreuche J and Susen S. Pulmonary Embolism in COVID-19 Patients: Awareness of an Increased Prevalence.Circulation . 0 .

4. DeYoung E and Minocha J. Inferior Vena Cava Filters: Guidelines, Best Practice, and Expanding Indications. Semin Intervent Radiol . 2016;33:65-70.

5. Uppal B, Flinn WR and Benjamin ME. The bedside insertion of inferior vena cava filters using ultrasound guidance. Perspect Vasc Surg Endovasc Ther . 2007;19:78-84.

6. Poyiadji N, Shahin G, Noujaim D, Stone M, Patel S and Griffith B. COVID-19-associated Acute Hemorrhagic Necrotizing Encephalopathy: CT and MRI Features. Radiology . 0:201187.

7. Konstantinides SV, Meyer G, Becattini C, Bueno H, Geersing G-J, Harjola V-P, Huisman MV, Humbert M, Jennings CS, Jiménez D, Kucher N, Lang IM, Lankeit M, Lorusso R, Mazzolai L, Meneveau N, Áinle FN, Prandoni P, Pruszczyk P, Righini M, Torbicki A, Van Belle E and Zamorano JL. 2019 ESC Guidelines for the diagnosis and management of acute pulmonary embolism developed in collaboration with the European Respiratory Society (ERS). The Task Force for the diagnosis and management of acute pulmonary embolism of the European Society of Cardiology (ESC) . 2019:1901647.

8. Tritschler T, Kraaijpoel N, Le Gal G and Wells PS. Venous Thromboembolism: Advances in Diagnosis and Treatment. Jama . 2018;320:1583-1594.

9. Marik PE, Andrews L and Maini B. The incidence of deep venous thrombosis in ICU patients. Chest . 1997;111:661-4.

\section{Legends:}

\section{Figure 1. Radiographic information and IVC filter implantation of patient 1.}

(A ) At day 6, X-ray radiography showed a blurred texture of bilateral lungs. (B ) At day 10, representative COVID-19 lesions could be seen in bilateral lungs. (C-D ) IVC filter implantation was successfully performed with the aid of DSA guiding. The IVC was patent. (E ) At day 13, there were fewer inflammatory lesions compared to day 6 (A ). (F ) At day 23, the follow-up CT scan showed fewer inflammatory lesions compared to the day $10 \mathrm{CT}$ image $(\mathbf{B})$.

\section{Figure 2. Bedside ultrasound-guided IVC filter implantation of patient 2 on the isolation ward.}

(A-B ) Severe ischemia signs were observed in the right thigh and left toes. (C) There was no blood flow in the right iliac artery and vein. (D) The tip of the IVC filter sheath was adjusted to be $1 \mathrm{~cm}$ below the right renal vein. (E) One retrievable inferior vena cava filter was implanted. (F ) At day 3, after emergent right 
thigh amputation surgery, X-ray radiography showed obvious inflammatory lesions in bilateral lungs. There was no shift of the inserted IVC filter. (G ) At day 9, in the intensive care unit, bedside X-ray radiography presented exacerbation in bilateral lungs. Black arrow indicates the inferior vena cava filter.

\section{Figure 3. Radiographic information and bedside IVC filter implantation of pati-} ent 3 .

(A-B ) At day 3, a CT scan showed representative COVID-19 lesions in transverse and coronal windows, respectively. (C-D ) X-ray radiography presented the compressed fractures in the 12th thoracic vertebrae (T12) and the 1st lumbar (L1) vertebrae. (E ) There was no blood flow in the left iliac vein. (F-G ) After adjusting the tip of the IVC filter sheath, one retrievable inferior vena cava filter was implanted $1 \mathrm{~cm}$ below the right renal vein. (H-I ) At day 9, X-ray radiography showed successful internal fixation surgery. There was no shift of the inserted IVC filter. (J-K ) At day 18, there were obviously fewer inflammatory lesions in transverse and coronal windows compared to day 4 (C-D ), respectively. Black arrow indicates the inferior vena cava filter. 
Table 1. Continuous laboratory assays of patient 1.

Table 2. Continuous laboratory assays of patient 2.

Table 3. Continuous laboratory assays of patient 3.
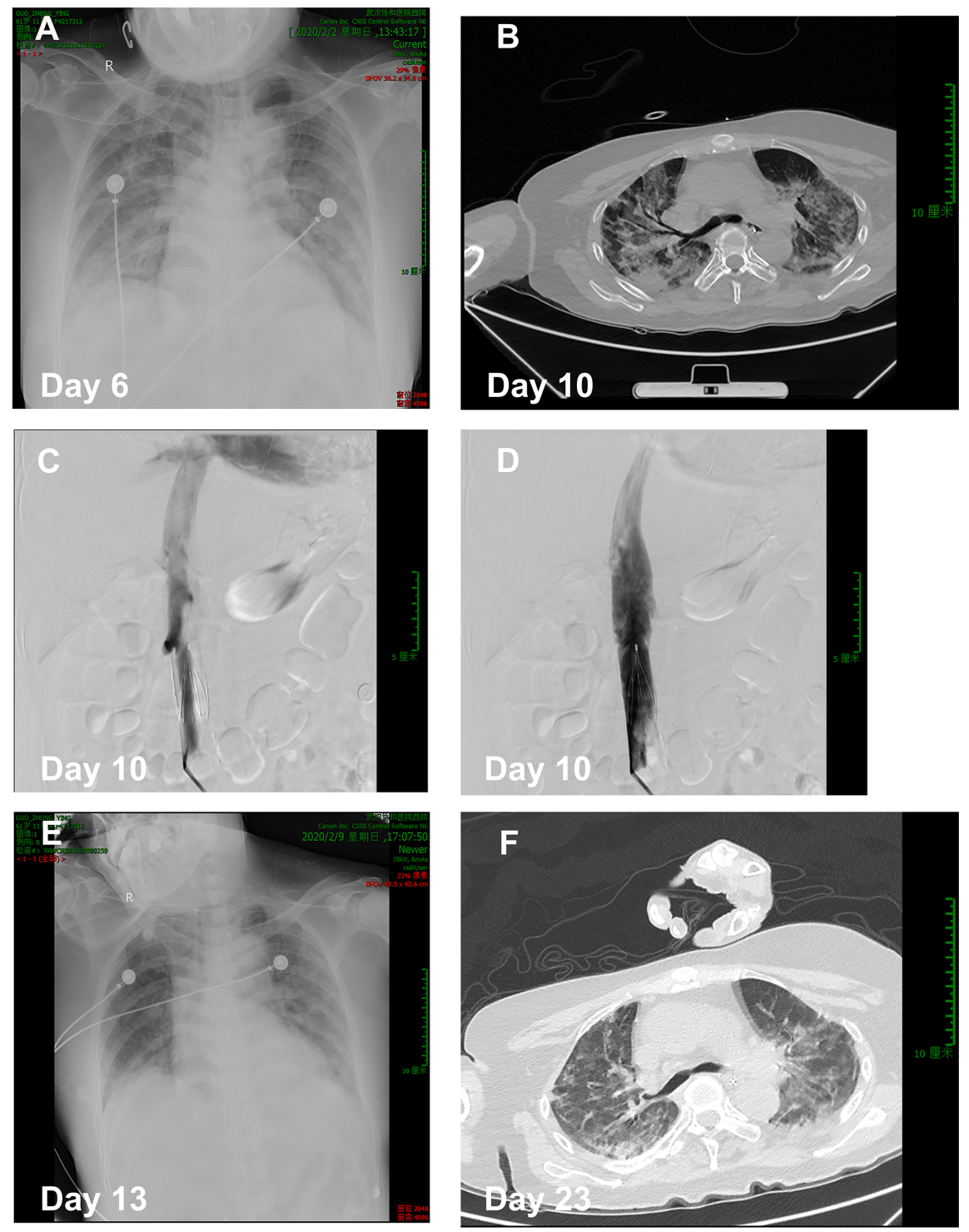

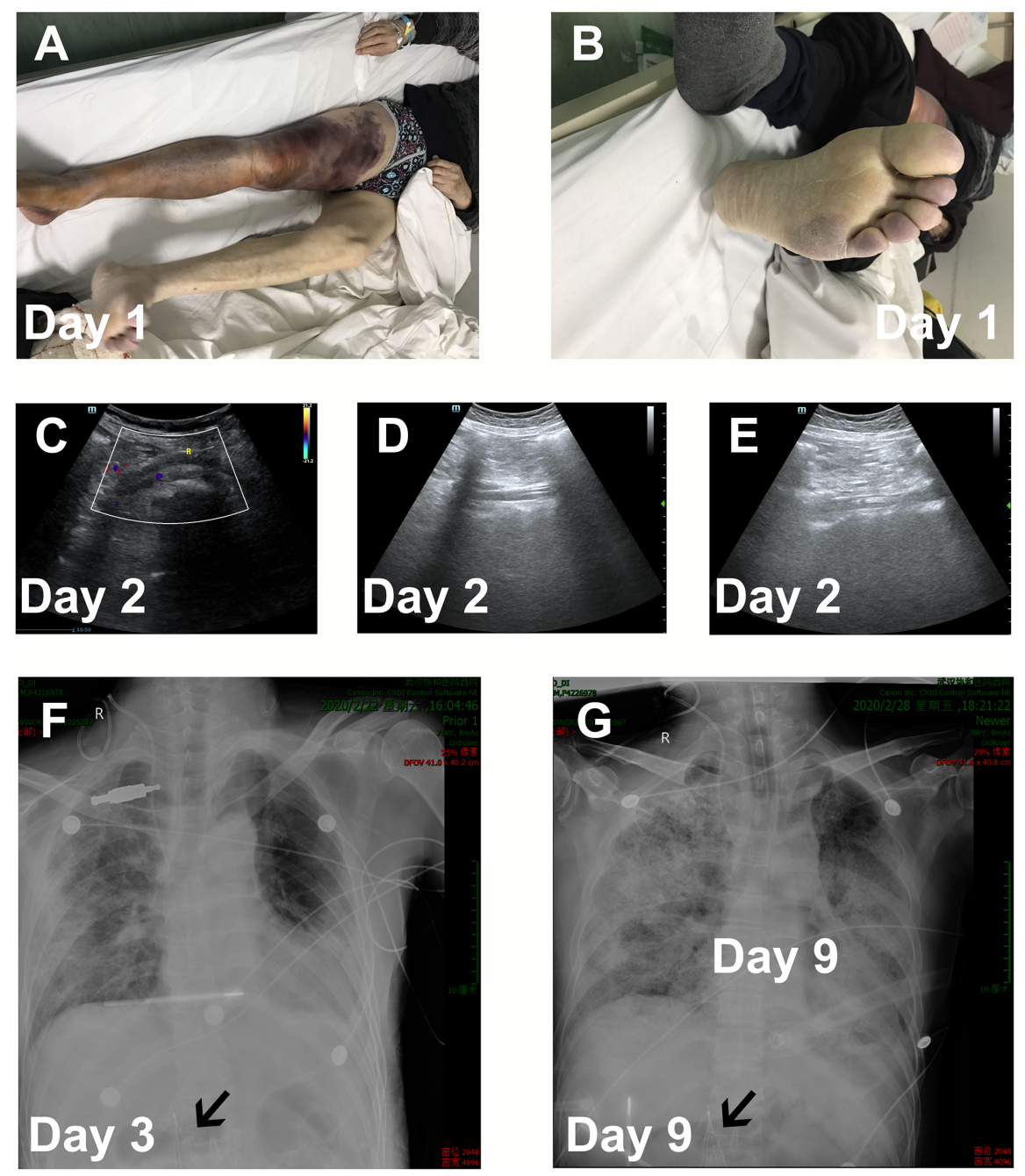

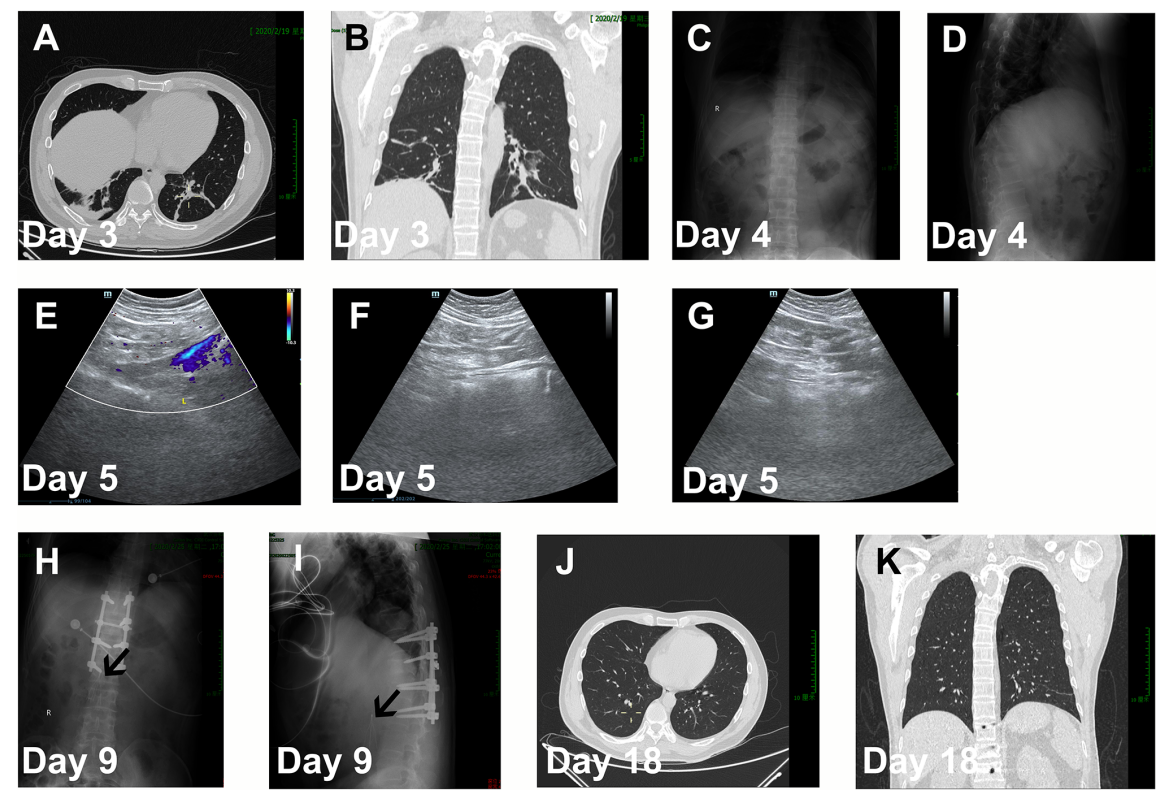